\title{
Chronic Pain: Lost Inhibition?
}

\author{
Luke A. Henderson, ${ }^{1}$ Chris C. Peck, ${ }^{2,5}$ Esben T. Petersen, ${ }^{3}$ Caroline D. Rae, ${ }^{4}$ Andrew M. Youssef,,${ }^{1}$ Jenna M. Reeves, ${ }^{1}$ \\ Sophie L. Wilcox, ${ }^{1}$ Rahena Akhter, ${ }^{2}$ Greg M. Murray, ${ }^{2}$ and Sylvia M. Gustin ${ }^{1}$ \\ ${ }^{1}$ Department of Anatomy and Histology and ${ }^{2}$ Faculty of Dentistry, University of Sydney, Sydney 2006, New South Wales, Australia, ${ }^{3}$ Departments of \\ Radiology and Radiotherapy, University Medical Center Utrecht, 3584 CX Utrecht, The Netherlands, ${ }^{4}$ Neuroscience Research Australia, Randwick 2031, \\ New South Wales, Australia, and ${ }^{5}$ School of Dentistry and Health Sciences, Charles Sturt University, Orange, New South Wales 2800 , Australia
}

Human brain imaging has revealed that acute pain results from activation of a network of brain regions, including the somatosensory, insular, prefrontal, and cingulate cortices. In contrast, many investigations report little or no alteration in brain activity associated with chronic pain, particularly neuropathic pain. It has been hypothesized that neuropathic pain results from misinterpretation of thalamocortical activity, and recent evidence has revealed altered thalamocortical rhythm in individuals with neuropathic pain. Indeed, it was suggested nearly four decades ago that neuropathic pain may be maintained by a discrete central generator, possibly within the thalamus. In this investigation, we used multiple brain imaging techniques to explore central changes in subjects with neuropathic pain of the trigeminal nerve resulting in most cases (20 of 23 ) from a surgical event. Individuals with chronic neuropathic pain displayed significant somatosensory thalamus volume loss (voxel-based morphometry) which was associated with decreased thalamic reticular nucleus and primary somatosensory cortex activity (quantitative arterial spin labeling). Furthermore, thalamic inhibitory neurotransmitter content was significantly reduced (magnetic resonance spectroscopy), which was significantly correlated to the degree of functional connectivity between the somatosensory thalamus and cortical regions including the primary and secondary somatosensory cortices, anterior insula, and cerebellar cortex. These data suggest that chronic neuropathic pain is associated with altered thalamic anatomy and activity, which may result in disturbed thalamocortical circuits. This disturbed thalamocortical activity may result in the constant perception of pain.

\section{Introduction}

Human brain imaging investigations have led to the idea that pain results from the coherent activation in multiple brain regions (Melzack, 1999). Although this appears true for acute pain, similar activation patterns do not occur in individuals with chronic pain, i.e., $>3$ months duration (Di Piero et al., 1991; Hsieh et al., 1995; Iadarola et al., 1995). Instead, pain resulting from nervous system damage (neuropathic pain) is consistently associated with ongoing thalamic activity decreases on the side contralateral to the ongoing pain (Moisset and Bouhassira, 2007) and altered thalamocortical rhythm (Gücer et al., 1978; Llinás et al., 1999; Sarnthein et al., 2006; Stern et al., 2006; Walton and Llinás, 2010), which raises the idea that chronic pain may result from the misinterpretation of altered thalamocortical coherence (Yezierski, 1996).

Consistent with this idea, it has been hypothesized that neuropathic pain is maintained by altered activity within a discrete brain region, i.e., a central generator (Head and Holmes, 1911; Melzack and Loeser, 1978; Latremoliere and Woolf, 2009; von

Received Jan. 14, 2013; revised March 15, 2013; accepted March 18, 2013.

Author contributions: L.A.H., C.C.P., G.M.M., and S.M.G. designed research; L.A.H., C.C.P., S.L.W., R.A., G.M.M., and S.M.G. performed research; E.T.P. and C.D.R. contributed unpublished reagents/analytic tools; L.A.H., C.D.R., A.M.Y., J.M.R., S.L.W., and S.M.G. analyzed data; L.A.H., C.C.P., G.M.M., and S.M.G. wrote the paper.

This work was supported by funding from the Australian National Health and Medical Research Council. We are grateful to Richard A.E. Edden for provision of the software patch for MEGA-PRESS, enabling measurement of GABA. The authors declare no competing financial interests.

Correspondence should be addressed to Dr. Luke A. Henderson, Department of Anatomy and Histology, F13, University of Sydney, Sydney 2006, NSW, Australia. E-mail: Iukeh@anatomy.usyd.edu.au.

DOI:10.1523/JNEUROSCI.0174-13.2013

Copyright $\odot 2013$ the authors $\quad 0270-6474 / 13 / 337574-09 \$ 15.00 / 0$
Hehn et al., 2012). Indeed, multiple lines of evidence suggest a critical role for the thalamus in chronic pain processing. In humans, thalamic activation can evoke painful sensations (Lenz et al., 1993; Davis et al., 1996); lesions encompassing the somatosensory thalamus [ventroposterior nucleus (VP)] can result in persistent neuropathic pain (Kim et al., 2007; Klit et al., 2009; Hong et al., 2010); and individuals with neuropathic pain have reduced thalamic volumes and biochemical changes indicative of neuronal loss (Pattany et al., 2002; Apkarian et al., 2004; Gustin et al., 2011) and display more frequent bursting activity of VP thalamic neurons (Hirayama et al., 1989; Lenz et al., 1989, 1998; Gerke et al., 2003). These thalamic alterations may result in the development of thalamocortical dysrhythmia.

Thalamocortical rhythm involves the processing of recurrent thalamic circuits of which the thalamic reticular nucleus (TRN) plays a significant role (Pinault, 2004). Cortically projecting VP thalamic neurons send collaterals to the TRN which in turn sends inhibitory GABA inputs back to the VP thalamus (Lam and Sherman, 2011). If neuropathic pain is associated with altered VPcortical outputs, a reduction in TRN activity may develop, resulting in reduced GABAergic input to VP and ultimately altered thalamocortical rhythm, which may result in the persistent perception of pain (Yezierski, 1996).

In this investigation, we used magnetic resonance imaging to explore thalamic function in subjects with painful trigeminal neuropathy. Although many previous studies have reported volumetric changes in chronic pain populations (Apkarian et al., 2004; Schmidt-Wilcke et al., 2007; Schweinhardt et al., 2008; Younger et al., 2010; Moayedi et al., 2011), few have explored 
individuals with trigeminal neuropathic pain. In this study, voxel-based morphometry (VBM), quantitative arterial spin labeling, magnetic resonance spectroscopy, and resting and stimulus-evoked functional MRI (fMRI) were used to explore thalamic anatomy, biochemistry, activity, and connectivity in individuals with neuropathic pain. We hypothesize that neuropathic pain will be associated with VP thalamus volume loss, decreased TRN blood flow, decreased thalamic GABAergic content, and altered thalamocortical connectivity.

\section{Materials and Methods}

\section{Subjects}

Twenty-three subjects with painful trigeminal neuropathy [(PTN); 19 females, mean age $49.8 \pm 1.7$ years $( \pm$ SEM $)]$ and 43 pain-free controls (31 females, mean age $49.8 \pm 2.3$ years) were recruited for the study. There was no significant difference in age $(t$ test; $p>0.05)$ or gender composition ( $\chi^{2}$ test, $p>0.05$ ) between the two subject groups. PTN subjects were diagnosed using the Liverpool criteria (Nurmikko and Eldridge, 2001). During the $7 \mathrm{~d}$ before the MRI session, each subject kept a pain diary, recording three times a day the intensity of their ongoing pain. Subjects rated the intensity of their pain using a $10 \mathrm{~cm}$ horizontal visual analog scale (VAS) with zero indicating "no pain" and 10 indicating "the most intense imaginable pain." These pain intensity scores were then averaged over the $7 \mathrm{~d}$ period to create a mean "diary" pain intensity score. On the day of the MRI scanning, subjects also rated their pain on a $10 \mathrm{~cm}$ VAS, i.e., "scan" pain. Diary pain was used to determine relationships between pain and the long-term brain measure of gray-matter volume. Scan pain was used to determine relationships between pain and shortterm measures, such as ongoing regional neural activity, magnetic resonance spectroscopy, and resting-state fMRI. During each scanning session, each subject also drew the distribution of their ongoing pain and completed a McGill pain questionnaire. Informed written consent was obtained for all procedures according to the Declaration of Helsinki and the study was approved by our local Institutional Human Research Ethics Committees. Some of the subjects used in this study were also used in previous investigations (Gustin et al., 2011, 2012).

\section{MRI acquisition}

All 23 PTN and 43 control subjects lay supine on the bed of a Philips Acheiva 3T MRI scanner (Philips Medical Systems) with their head immobilized in a tight-fitting head coil. In each subject, three highresolution 3D T1-weighted anatomical image sets, covering the entire brain, were collected (turbo-field echo; echo time, $2.5 \mathrm{~ms}$; repetition time, $5600 \mathrm{~ms}$; flip angle, $8^{\circ}$ voxel size, $0.8 \times 0.8 \times 0.8 \mathrm{~mm}$ ). Three acquisitions were acquired to improve signal-to-noise ratios. In 18 of 23 PTN and 29 of 43 control subjects (PTN: 14 females, mean age $49.4 \pm 2.0$ years; controls: 17 females, mean age $50.1 \pm 2.9$, no significant difference in age or gender), a quantitative arterial spin labeling (QASL) series, encompassing the entire brain, was then collected $[\mathrm{TR} / \mathrm{TE} / \mathrm{DTI} / \mathrm{TI} 1=$ 4000/23/300/40 ms, $64 \times 64$ matrix, 14 slices; FOV, $240 \times 240$; flip angle, $35 / 11.7^{\circ} ;$ SENSE $=2.5 ; V_{\text {enc }}=[\infty, 4 \mathrm{~cm} / \mathrm{s}], 82\left(48\right.$ at $V_{\text {enc }}=4 \mathrm{~cm} / \mathrm{s}, 24$ at $V_{\text {enc }}=\infty, 10$ low flip-angle), all implemented in two separate sequences] (Petersen et al., 2006). In addition, a series of T1-weighted anatomical images was collected at the same slice locations as the QASL images (echo time, $2.5 \mathrm{~ms}$; repetition time, $5600 \mathrm{~ms}$; flip-angle, $8^{\circ} ; 256 \times 256$ matrix, 14 slices, FOV $=240 \times 240)$. In 14 PTN subjects and 17 controls $(\mathrm{PTN}$ : 12 females, mean age $50.5 \pm 2.8$; controls: 10 females, mean age $43.8 \pm$ 2.6 , no significant difference in age or gender), thalamic organization was assessed by collecting multiple series of 130 gradient-echo echoplanar fMRI image volumes using blood oxygen level-dependent contrast. Each image volume contained 43 axial slices covering the entire brain (voxel = $1.95 \times 1.95 \times 3.00 \mathrm{~mm}$ thick; repetition time, $3000 \mathrm{~ms}$; echo time, 40 $\mathrm{ms}$ ). During each $\mathrm{fMRI}$ series, the lateral part of the lower lip was brushed with a plastic brush at $\sim 2$ strokes/s. This stimulation paradigm was performed for a period of $10 \mathrm{fMRI}$ volumes $(30 \mathrm{~s})$ following a baseline period of $10 \mathrm{fMRI}$ volumes $(30 \mathrm{~s})$. This was repeated a further five times for a total of six stimulation and seven baseline periods. In control subjects, only the right side of the body was brushed. In all PTN subjects, the side ipsilateral to the highest ongoing pain was brushed.
Fourteen of 23 PTN and 30 of 43 controls subjects (PTN: 11 females, mean age $49.1 \pm 2.5$; controls: 19 females, mean age $46.6 \pm 2.4$, no significant difference in age or gender) either continued their initial scanning session (controls, $n=25$, PTN, $n=6$ ) or returned up to 12 months later for a second MRI session (controls, $n=5$, PTN, $n=8$ ). In the PTN subjects who returned for a second MRI session, pain intensity was assessed at the time of scanning, and pain duration was determined and the values of each of these were adjusted in the appropriate analyses. During this session GABA-edited MEGA-PRESS spectroscopy (Mescher et al., 1998; Edden and Barker, 2007) was performed on the thalamus contralateral to the pain in PTN subjects and on the right side in control subjects. An isotropic 3D scan with multiplanar (axial, sagittal, coronal) reformats covering the whole brain was acquired (turbo-field echo; echo time, $2.5 \mathrm{~ms}$; repetition time, $5600 \mathrm{~ms}$; flip angle, $8^{\circ}$; voxel size, $0.8 \times$ $0.8 \times 0.8 \mathrm{~mm})$ for voxel placement $(20 \times 20 \times 20 \mathrm{~mm})$ (see Fig. 3$)$. The MEGA-PRESS sequence parameters were as follows: repetition time, $2000 \mathrm{~ms}$; echo time, $68 \mathrm{~ms}$; 1024 acquisition points; total acquisition time, $27 \mathrm{~min}$. One hundred averages were acquired with the MEGAPRESS editing pulse centered at $1.9 \mathrm{ppm}$ ("ON spectra") and 100 averages with the pulse centered at $7.6 \mathrm{ppm}$ ("OFF spectra"). Automatic shimming (pencil beam auto first-order option) was performed resulting in line widths of $<10 \mathrm{~Hz}$ for all spectra.

In addition, in 12 PTN subjects and 20 controls, a series of 180 gradient-echo echoplanar fMRI image volumes using blood oxygen level-dependent contrast were collected. Each image volume contained 35 axial slices covering the entire brain (voxel, $3 \times 3 \times 4 \mathrm{~mm}$ thick; repetition time, $2000 \mathrm{~ms}$; echo time, $30 \mathrm{~ms}$ ). During this fMRI series, each PTN subject was instructed to remain relaxed, and no stimulation paradigm was performed. To functionally identify the medial division of the VP nucleus (VPM) thalamus, a 180 volume fMRI series was collected during which the right corner of the lower lip was lightly stroked at $\sim 2$ strokes/s using a plastic brush. This stimulation paradigm was performed for a period of $20 \mathrm{fMRI}$ volumes ( $40 \mathrm{~s}$ ) following a baseline period of 20 fMRI volumes ( $40 \mathrm{~s}$ ). This was repeated a further three times for a total of four stimulation and five baseline periods.

\section{MRI analysis}

Gray-matter volume. Using SPM8 (Friston et al., 1995), the three T1weighted images from each subject were coregistered and averaged. The averaged image was bias corrected using the SPM8 unified segmentation (Ashburner and Friston, 2005). The bias-corrected images were segmented and spatially normalized using a second pass of the unified segmentation algorithm. The result of the segmentation and spatial normalization were whole-brain "maps" of gray-matter probabilities, spatially normalized into the Montreal Neurological Institute (MNI) template space, and "modulated" by the volume changes due to the normalization. The normalized, modulated gray-matter images were then smoothed using a $6 \mathrm{~mm}$ full-width-at-half-maximum (FWHM; 6 $\mathrm{mm}$ ) Gaussian filter. In 11 PTN subjects, the ongoing pain was localized either exclusively or predominantly to one side of the face (Table 1). That is, on one side of the face, pain intensity was either zero or at least $75 \%$ lower than the pain intensity on the opposite side of the face. For PTN subjects with right-sided pain $(n=6)$, the T1-image sets used for VBM analysis were reflected in the $x$-plane (i.e., to the opposite side) so that each subject's brain then represented pain on the same side of the face (i.e., the left side). In this manner, we were able to assess gray-matter volume changes ipsilateral and contralateral to the ongoing pain.

Significant differences in gray matter between PTN and control subjects were determined using random-effects analysis with age, sex, and total brain volume as nuisance variables $(p<0.01$, false discovery rate corrected for multiple comparisons). Significant gray-matter volume differences were then overlaid onto an individual's T1-weighted image. Within the contralateral thalamus, significant gray-matter volumes were extracted for PTN and control subjects in the region of overlap between gray-matter loss and brushing activation (see below for brushing analysis) and compared using a two-sample $t$ test ( $p<0.05$ set as significant). Further, in PTN subjects, correlations between gray-matter volume and diary pain intensity and duration were determined $(p<0.05$, Bonferroni corrected for multiple comparisons). Finally, PTN subjects were grouped 
Table 1. Characteristics of patients with PTN (neuropathic pain)

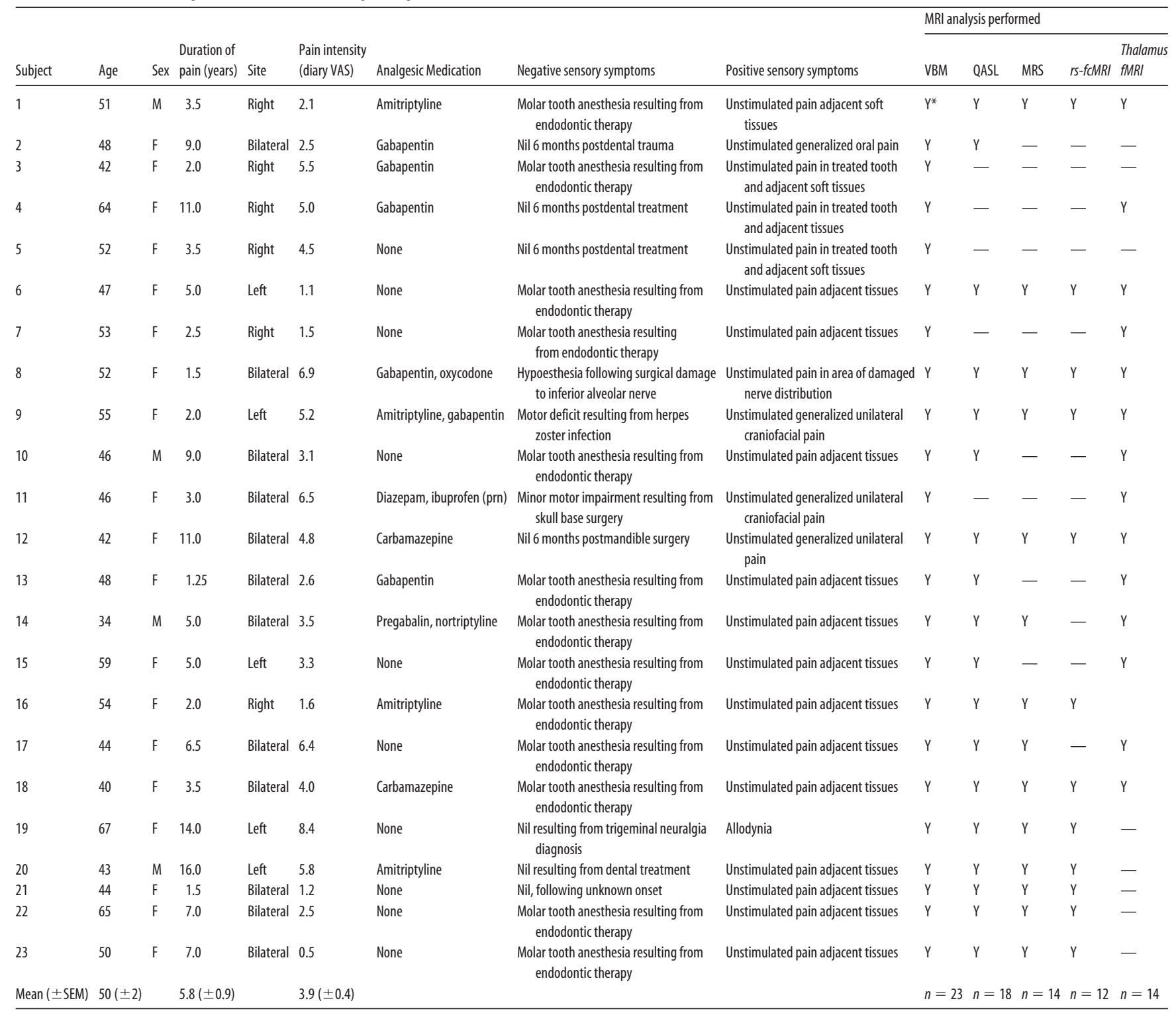

* $Y$ indicates subject was included in a particular MRI analysis.

PRN, Pro re nata (as needed); rs-fcMRl, resting-state functional connectivity magnetic resonance imaging. Note: IASP definition, Neuropathic pain is a clinical description (and not a diagnosis) which requires a demonstrable lesion or a disease that satisfies established neurological diagnostic criteria. The term "lesion" is commonly used when diagnostic investigations reveal an abnormality or when there was obvious trauma. The term "disease" is commonly used when the underlying cause of the lesion is known. The presence of symptoms or signs (e.g., touch-evoked pain) alone does not justify the use of the term "neuropathic." It is common when investigating neuropathic pain that diagnostic testing may yield inconclusive or even inconsistent data. In such instances, clinical judgment is required to reduce the totality of findings in a patient into one putative diagnosis or concise group of diagnoses.

into those taking analgesic medications $(n=13)$ and those not taking any medications $(n=10)$, and gray-matter volumes between these two groups were compared using a two-sample $t$ test $(p<0.05$ set as significant).

Thalamic organization. All fMRI images were motion corrected, and global signal drifts were removed using the detrending method described by Macey et al. (2004), spatially normalized to the MNI template, and smoothed using a $4 \mathrm{~mm}$ FWHM Gaussian filter. Significant increases in signal intensity were determined using a repeated boxcar model convolved with hemodynamic delay function (10 volumes baseline, 10 volumes lip brushing; 6 stimulation, and 7 baseline periods). Significant activation within the contralateral thalamus was determined using a random-effects analysis ( $p<0.05$, false discovery rate corrected for multiple comparisons, minimum cluster size 10 voxels). The most significantly activated voxel was then used to create an 8 -mm-radius sphere as a region of interest. In each individual control and PTN subject, the maximally activated voxel within this sphere was then determined and the $x, y$, and $z$ coordinates were compared between control and PTN groups (two-sample $t$ test, $p<0.05$ ). Furthermore, in each subject, percent signal intensity changes (relative to baseline) for each of the 130 volumes was extracted from a $2-\mathrm{mm}$-radius sphere at this location. The percentage change in signal intensity was calculated for each brushing period relative to the initial baseline period. Significant differences in signal intensity changes between controls and PTN subjects were assessed (two-sample $t$ test, $p<0.05$ ).

Cerebral blood flow. QASL images were opened using custom software and cerebral blood flow (CBF) maps were created (Petersen et al., 2006). In addition, from the CBF maps, anatomical (gray/white) image sets were created. These anatomical images were then coregistered to the T1weighted anatomical image set collected at the same slice locations, and the resulting parameters were applied to the CBF maps. The T1-weighted anatomical images were then normalized to the standard MNI template and the normalization parameters were applied to the CBF maps. The resulting spatially normalized CBF maps were then smoothed $(6 \mathrm{~mm}$ FWHM Gaussian filter). For PTN subjects with right-sided pain $(n=2)$, the CBF maps were reflected in the $x$-plane (i.e., to the opposite side), so 
A

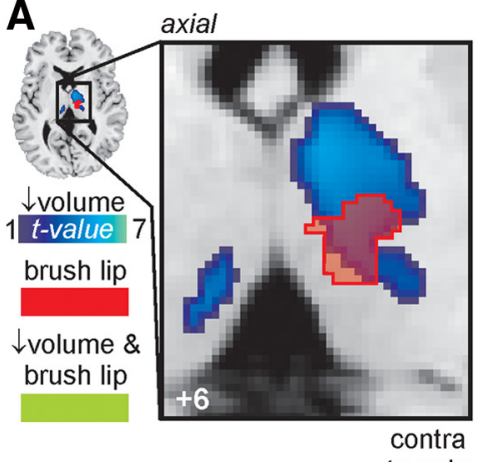

to pain
B

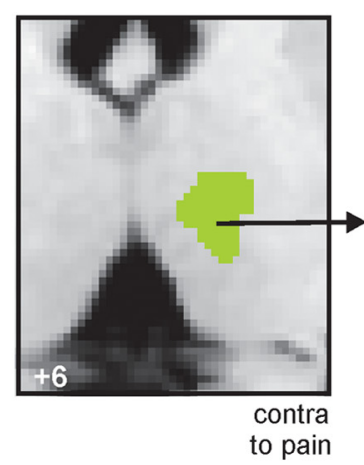

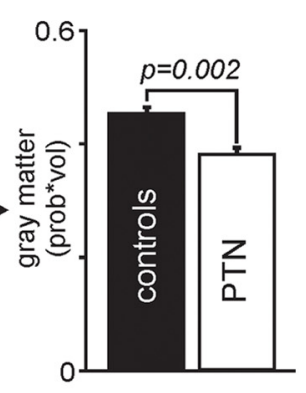

Figure 1. A, Gray-matter volume decreases (cool color scale) in the thalamus of subjects with PTN compared with pain-free controls overlaid onto an individual's axial T1-weighted anatomical image. Thalamic region activated during innocuous lip brushing in 20 control subjects is indicated by the red shading and its overlap with gray-matter loss by the green shading. This green shading encompasses the somatosensory thalamus, i.e., ventroposterior nucleus. The slice location in MNI space is indicated at the bottom-left of the image. $\boldsymbol{B}$, Plots of mean ( \pm SEM) gray-matter volumes in the somatosensory thalamus in PTN subjects and pain-free controls.
A

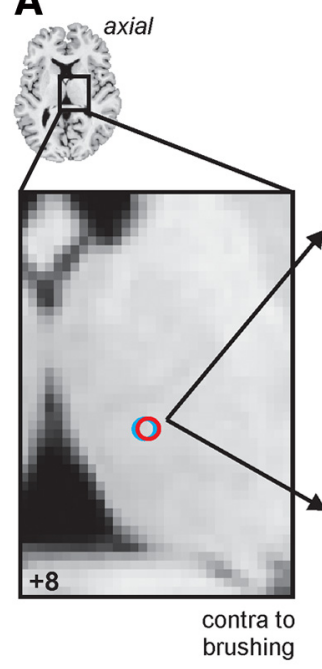

B

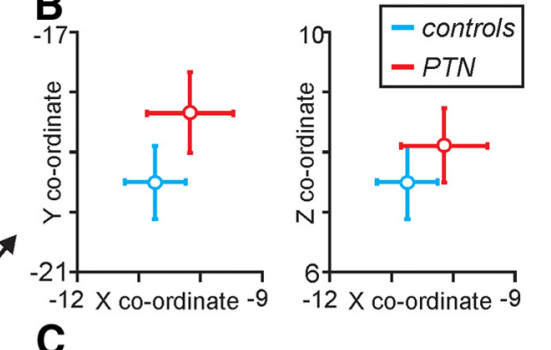

C

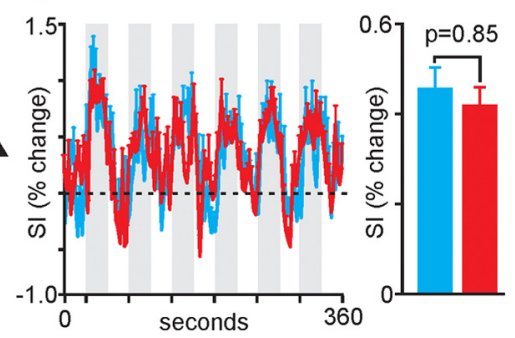

Figure 2. A, Thalamic activation during innocuous brushing of the lip in 20 control and 12 subjects with PTN displayed on an individual's axial T1-weighted anatomical image. $\boldsymbol{B}$, Plots of mean ( \pm SEM) $x, y$, and $z$ coordinates in MNI space during lip brushing in controls (blue) and PTN subjects (red). C, plots of mean ( \pm SEM) percentage signal intensity (SI) change during lip brushing in controls and PTN subjects. The vertical gray bars represent the brushing periods. The bar graph shows the mean ( \pm SEM) percentage change during all lip brushing periods in controls and PTN subjects. Note that neither the location nor magnitude of signal intensity change differed between controls and PTN subjects.

that each subject's brain then represented pain on the same side of the face. Significant differences in CBF between PTN and control subjects were determined using random-effects analysis with age and sex as nuisance variables. Given that we hypothesized that thalamic and primary somatosensory cortex (SI) regions would display altered blood flow, we used standard regions of interest derived from the MNI PickAtlas toolbox in SPM8, to apply small volume corrections $(p<0.05$, false discovery rate corrected for multiple comparisons). Significant CBF differences were then overlaid onto an individual's T1-weighted image.

CBF values from significant clusters were extracted from both the PTN and control subjects and compared using two-sample $t$ tests $(p<0.05$ set as significant). In PTN subjects, correlations between CBF and scan pain intensity and duration were determined $(p<0.05$, Bonferroni corrected for multiple comparisons). In addition, to compare CBF within the VP thalamus of control and PTN subjects, CBF values were extracted from the region of overlap between gray matter loss and brushing activation (see below for brushing analysis) and compared using a two-sample $t$ test ( $p<0.05$ set as significant). Finally, PTN subjects were grouped into those taking analgesic medications $(n=10)$ and those not taking any medications $(n=8)$, and CBF values between these two groups were compared using a two-sample $t$ test $(p<0.05$ set as significant).

GABA-edited MEGA-PRESS spectra. For quantification of the GABA/creatine ratios, the acquired spectra were analyzed using the Javabased magnetic resonance user's interface (jMRUI 4.0, European Union project). First, the dominant water resonance was removed using the Hankel Lanczos Singular Valve Decomposition algorithm. The "ON" and "OFF" spectral subsets were summed producing single $\mathrm{ON}$ and OFF 68 ms subspectra for each spectra dataset. These $68 \mathrm{~ms}$ subspectra were then subtracted resulting in GABA-edited difference spectra to measure GABA concentration at $3.01 \mathrm{ppm}$. The GABA-edited difference spectra were then phased with respect to both the zero-order and first-order phase. Line broadening of $5 \mathrm{~Hz}$ was used to improve the display. GABA was quantified using AMARES, a nonlinear least-square fitting algorithm operating in the time domain. Peak fitting for GABA was performed after manually defining the center frequency and line width of the GABA peak and modeling the GABA peak as a singlet. Furthermore, Lorentzian curves were used to obtain the peak amplitude for this resonance.

The OFF spectral subsets were summed producing single OFF $68 \mathrm{~ms}$ subspectra for each spectra dataset to measure creatine concentration at $3.02 \mathrm{ppm}$. The single OFF $68 \mathrm{~ms}$ subspectra was then phased with respect to both the zero-order and first-order phase. Line broadening of $5 \mathrm{~Hz}$ was used to improve the display. Spectral fitting in AMARES was performed after manually defining the center frequency and line width of the creatine peak, and modeling the creatine peak as a singlet. Furthermore, Lorentzian curves were used to obtain the peak amplitude for this resonance. Finally, ratios were calculated for GABA relative to creatine.

Differences in metabolite ratios between PTN and control subjects were determined using two-sample $t$ tests ( $p<0.05$ set as significant). In PTN subjects, correlations between thalamic GABA/Cr (creatine) and scan pain intensity and duration were determined $(p<0.05$, Bonferroni corrected for multiple comparisons). Finally, thalamic GABA/Cr ratios in PTN subjects taking analgesic medications $(n=8)$ were compared with PTN subjects not taking analgesic medications $(n=6)$ using a two-sample $t$ test ( $p<0.05$ set as significant).

Functional connectivity. All fMRI images were motion-corrected, global signal drifts removed using the detrending method described by Macey et al. (2004), spatially normalized to the MNI template. To functionally identify the location of the VPM thalamus, fMRI images collected during lip brushing were smoothed using a $6 \mathrm{~mm}$ FWHM Gaussian filter, and significant increases in signal intensity were determined using a repeated boxcar model convolved with hemodynamic delay function (20 volumes baseline, 20 volumes lip brushing; 4 stimulation, and 5 baseline periods). Significant activation within the contralateral thalamus was determined using a random-effects analysis $(p<$ 0.05 , false discovery rate corrected for multiple comparisons, minimum cluster size 10 voxels). This cluster was then used in the VBM analysis to determine significant changes in gray-matter volume in PTN subjects compared with controls.

For connectivity analysis, images were smoothed using a $6 \mathrm{~mm}$ FWHM Gaussian filter. In all subjects, during the baseline fMRI scan (i.e., no stimulation paradigm), functional connectivity was assessed using signal intensity changes within the VPM thalamus as a "seeding area." More specifically, signal intensity changes over the 180 volumes were extracted in each subject from the thalamic region activated by lip brushing and also displaying gray-matter loss in PTN subjects (i.e., VPM thal- 
amus). For PTN subjects with right-sided pain $(n=2)$, the fMRI images were reflected in the $x$-plane (i.e., to the opposite side) so that each subject's brain then represented pain on the same side of the face. VPM signal intensity was then entered as a regressor into a first-level analysis, and brain regions in which signal intensity matched each individual subject's VPM signal intensity were determined. The six movement parameters derived from the motion correction step were entered as nuisance variables to factor out any movement related signal intensity changes. The resulting contrast maps were then entered into a second-level analysis, and comparisons between resting connectivity in controls and PTN subjects were determined ( $p<0.05$, false discovery rate corrected for multiple comparisons). In addition, each individual subject's thalamus GABA/creatine ratios were entered as a regressor and the relationship between thalamic GABA/creatine and VPM connectivity was then determined in the control group and in the PTN group $(p<$ $0.05, r>0.70)$. In PTN subjects, contrast values were extracted from significant regions and plotted against thalamic GABA/creatine ratios and $r$ values, and significance was determined $(p<0.05$ set as significant). A significant negative correlation means that as thalamic GABA content decreases, signal intensity covaries more closely with VPM thalamic signal intensity. Finally, contrast values in regions that displayed significant correlations in PTN subjects were extracted from controls and plotted. Significant differences in these correlations between controls and PTN subjects were then assessed using Fisher $r$-to- $z$ transformation $(p<0.05)$.

\section{Results}

Individual and mean subject characteristics are shown in Table 1. On average, PTN patients had ongoing pain of $3.9 \pm 0.4$ of 10 , with an average duration of $5.8 \pm 0.9$ years. The five most common pain descriptors selected from the sensory component of the McGill pain questionnaire by PTN subjects were "aching," "sharp," "shooting," "gnawing," and "radiating."

\section{Thalamic gray-matter volume}

Using modulated VBM techniques, we found that after removing the effects of age, gender, and total brain size by adding them as nuisance variables, PTN patients had significantly reduced $(p<$ 0.05 , corrected for multiple comparisons) gray-matter volumes in the ipsilateral (to ongoing pain) anterior insula, primary somatosensory cortex, thalamus, and contralateral nucleus accumbens (PTN: $n=23$; controls: $n=43$ ). In addition, we found a significant volume decrease in the contralateral thalamus of PTN patients compared with controls (Fig. 1A). To determine whether this thalamic volume change encompassed the VPM (the nucleus that receives orofacial somatosensory information), we functionally identified the VPM nucleus in 20 control subjects using blood oxygen level-dependent fMRI. We found that the thalamic volume loss associated with chronic neuropathic pain did indeed mostly encompass the VPM thalamic nucleus defined by lip brushing, and this region of overlap displayed significantly lower gray-matter volumes in PTN subjects compared with controls [gray-matter volume $\times$ probability $( \pm$ SEM): controls, $0.46 \pm 0.01 ;$ PTN, $0.41 \pm 0.01 ; p=0.002$ ] (Fig. $1 B$ ). In PTN subjects, VPM volume was not significantly correlated to either
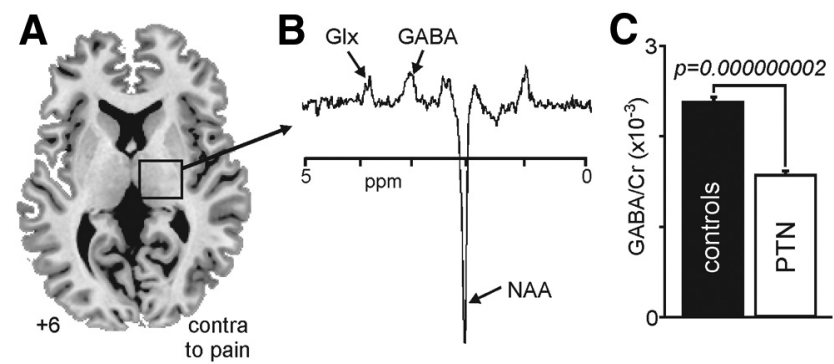

Figure 4. A, Axial slice showing location from which proton spectroscopy was performed in the contralateral thalamus of subjects with PTN and pain-free controls. Slice location in MNI space is indicated at the bottom-left of the image. $\boldsymbol{B}$, Typical MEGA-PRESS spectrum obtained from the thalamus. Glx, Glutamine; NAA, N-acetyl aspartate. C, A plot of mean ( \pm SEM) GABA/Cr ratio in the thalamus of PTN subjects and pain-free controls.

ongoing pain intensity $(r=-0.21, p=0.33)$ or pain duration $(r=0.001, p=0.98)$. Furthermore, there was no significant difference in thalamic gray-matter volumes in those PTN subjects taking analgesic medication compared with those not taking analgesic medication [gray-matter volume $\times$ probability $( \pm \mathrm{SEM})$ : medication, $0.40 \pm 0.02$; no medication, $0.42 \pm 0.02$; $p=0.14]$.

\section{Thalamic activation and organization}

Innocuous lip brushing in controls and PTN subjects resulted in similar activation patterns within the contralateral VP thalamus. There was no significant difference in the $x, y$, and $z$ coordinates of thalamic activation in controls compared with PTN subjects (mean $\pm \operatorname{SEM} x, y$, and $z$ coordinates: controls, $-10.8 \pm 0.5$, $-19.5 \pm 0.7,7.7 \pm 0.6 ; \mathrm{PTN},-10.1 \pm 0.8,-18.3 \pm 0.7,8.1 \pm$ 0.3 ; $p$ values: $x, p=0.47 ; y, p=0.23 ; z, p=0.59)$ (Fig. $2 B)$. In 


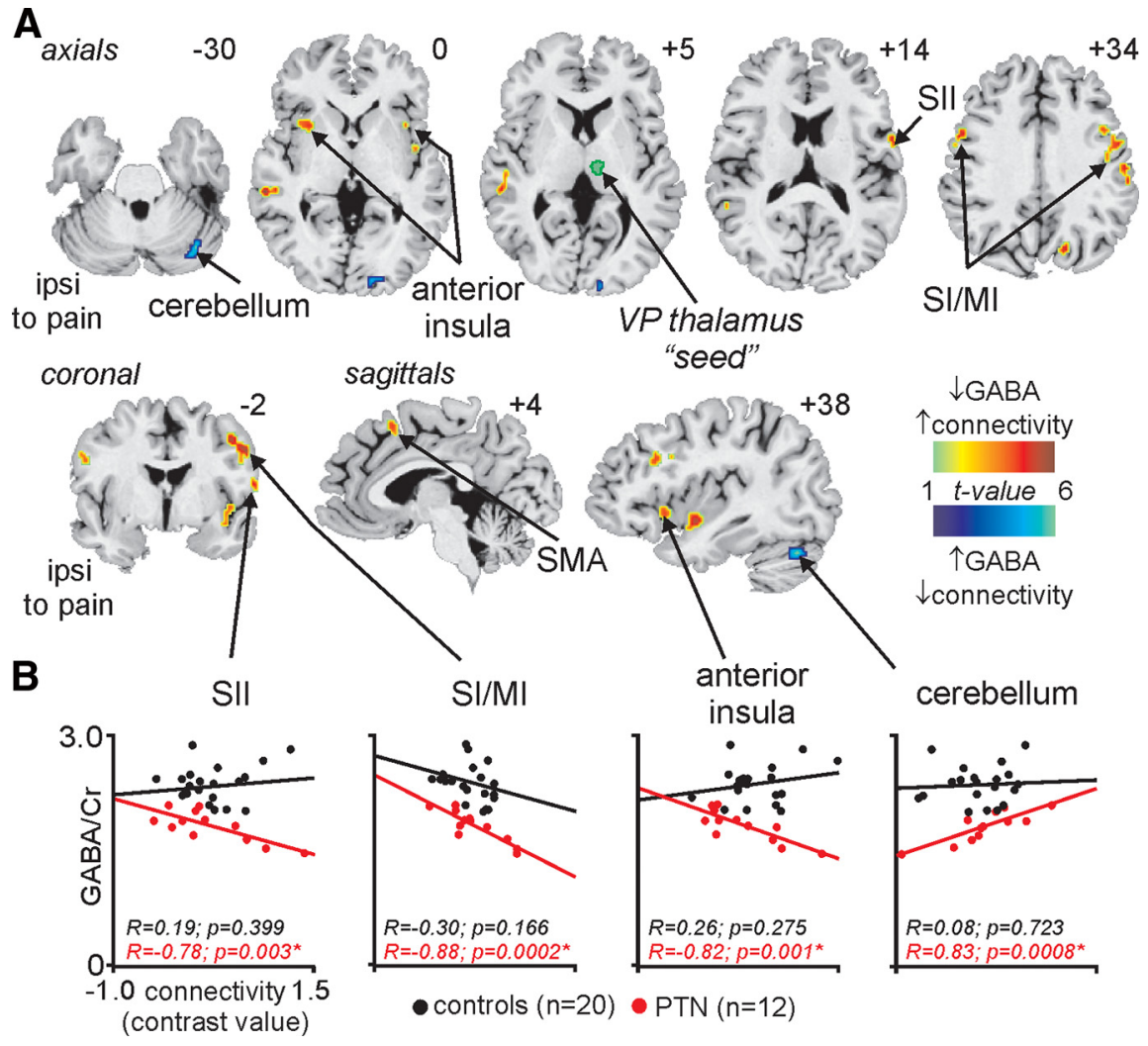

Figure 5. A, Brain regions in PTN subjects, in which the strength of the correlation between baseline signal fluctuations and signal within the somatosensory (ventroposterior thalamus) was significantly correlated with thalamic content. The ventroposterior thalamus "seed" region is indicated by the green shading. Significant clusters are overlaid onto an individual subject's T1weighted image set. The hot color scale indicates that as thalamic GABA content decreases, signal intensity fluctuates more closely with that of the ventroposterior thalamus. Slice locations in MNI space are indicated at the top-right of each image. MI, Primary motor cortex; PCC, posterior cingulate cortex; $\mathrm{SI}$, primary somatosensory cortex. $\boldsymbol{B}$, Plots of thalamic GABA/Cr values against connectivity strength in a number of brain regions in PTN subjects (red) and controls (black). Note that although in PTN subjects, thalamic GABA was significantly correlated to VP connectivity in a number of brain regions; it was not significantly correlated in controls. *Indicates significant differences between correlation strength in PTN and controls.

addition, there was no significant difference in the magnitude of fMRI signal intensity changes within the VP thalamus during lip brushing in controls compared with PTN subjects (mean \pm SEM percentage change: controls, $0.45 \pm 0.06$; PTN, $0.41 \pm 0.06 ; p=$ 0.85) (Fig. 2C).

\section{Thalamic and cortical perfusion}

Following removal of the effects of age and gender, we found that PTN patients had significantly reduced ( $p<0.05$, corrected for multiple comparisons) cerebral blood flow in a few discrete brain regions (PTN, $n=18$; controls, $n=29$ ). These included the contralateral (to the highest pain intensity) primary somatosensory cortex (S1) and thalamus. No significant change in thalamic or S1 cerebral blood flow occurred on the side ipsilateral to the ongoing pain. Within the contralateral thalamus, the CBF decrease was located on the lateral edge of the thalamus, encompassing the region of the thalamic reticular nucleus [mean CBF $\mathrm{ml} / \mathrm{min} / \mathrm{g}( \pm \mathrm{SEM}):$ controls, $27.8 \pm 1.7 ; \mathrm{PTN}, 19.5 \pm 1.3 ; p=$ 0.001 ] (Fig. 3A). More specifically, it was centered on the area of the TRN that has been shown to receive collaterals from VP neurons in monkeys (Pollin and Rokyta, 1982). In PTN subjects, TRN blood flow was negatively correlated to ongoing pain intensity $(r=-0.55, p=0.034)$; that is, the greater the reduction in TRN blood flow, the greater the ongoing pain (Fig. $3 A$ ). In contrast, TRN blood flow was not significantly correlated to pain dura- tion $(r=-0.05, p=0.82)$. PTN subjects also displayed a significant reduction in $\mathrm{CBF}$ within the orofacial region of the contralateral primary somatosensory cortex (S1; controls: $31.6 \pm 1.6$; PTN: $22.6 \pm 1.9 ; p=$ 0.0009) (Fig. 3B). In PTN subjects, S1 blood flow was not significantly correlated to either ongoing pain intensity $(r=0.18$; $p=$ $0.49)$ or pain duration $(r=-0.02 ; p=$ $0.92)$.

To determine whether there was a significant change within the VPM thalamus, we extracted CBF values from the thalamic region in which innocuous lip brushing overlapped with gray-matter volume decrease in PTN subjects (Fig. $1 A$ ). We found that blood flow within the VPM thalamus was not significantly different in PTN subjects compared with controls (controls, $38.0 \pm 2.3$; PTN, $37.9 \pm 3.3 ; p=0.98$ ) (Fig. $3 C$ ). Finally, there were no significant $\mathrm{CBF}$ differences in those PTN subjects taking analgesic medication compared with those not taking analgesic medication [mean CBF ml/ $\mathrm{min} / \mathrm{g}$ ( \pm SEM): TRN: medication, $19.3 \pm$ 1.9; no medication, $19.9 \pm 1.6 ; p=0.80$; SI: medication, $24.9 \pm 2.5$; no medication, $19.1 \pm 2.6 ; p=0.14]$.

\section{Thalamic GABA levels}

The thalamic region from which GABA spectra were collected as well as a typical spectrum are shown in Figure $4, A$ and $B$. After taking into account age and gender, PTN subjects had significantly lower GABA levels within the thalamus compared with controls [mean GABA/creatine ratio $( \pm \mathrm{SEM}) \times 10^{-3}$ : controls, $2.38 \pm 0.04$; PTN, $1.82 \pm$ 0.05; $p=0.000000002$; PTN, $n=14$; controls, $n=30$ ] (Fig. $4 C$ ). In PTN subjects, $\mathrm{GABA} /$ creatine ratios were not correlated to ongoing scan pain intensity $(r=-0.34, p=0.24)$ or pain duration $(r=0.31, p=0.28)$. Finally, there was no significant difference in thalamic GABA content in those PTN subjects taking analgesic medication compared with those not taking analgesic medication [mean GABA/creatine ratio $( \pm \mathrm{SEM}) \times 10^{-3}$ : medication, $1.80 \pm 0.06$; no medication, $1.85 \pm 0.10$; $p=0.65)$.

\section{Thalamocortical functional connectivity}

Using the contralateral (to ongoing pain) VPM region as a "seeding" area, we determined brain regions in which resting signal intensity fluctuated with signal intensity within the VPM thalamus, and then the relationship between thalamic GABA content and the degree of this functional connectivity was determined. We found no significant difference between controls and PTN subjects when we compared VP connectivity at rest. However, in 12 PTN subjects, thalamic GABA content was significantly negatively correlated to VPM functional connectivity within a number of brain regions (Fig. 5A). These included the ipsilateral (ipsi) and contralateral (to pain) (contra) primary somatosensory/motor cortex (ipsi, $r=-0.81, p=0.001$; contra, $r=-0.88$, $p=0.0002)$, contralateral secondary somatosensory cortex $(r=$ $-0.78, p=0.003)$, supplemental motor area $(r=-0.75, p=$ 
0.006 ), and the anterior insular cortex bilaterally (ipsi, $r=-0.75$, $p=0.006$; contra, $r=-0.82, p=0.001$ ) (Fig. $5 B$ ). In these brain regions, as thalamic GABA content decreased, ongoing signal intensity fluctuated more closely with signal intensity within the VPM thalamus. That is, in PTN subjects, the greater the thalamic GABAergic decrease, the greater the alteration in thalamocortical connectivity. In one region, the contralateral cerebellar cortex, as thalamic GABA content increased, ongoing signal intensity fluctuated more closely with signal intensity within the VPM thalamus $(r=0.83, p=0.0008)$.

In contrast, the only region that showed a significant correlation between VP functional connectivity and thalamic GABA in 20 controls was the medial prefrontal cortex. Furthermore, in regions that displayed significant correlations in PTN subjects, no such correlations occurred in controls (Fig. $5 B$ ). In addition, the correlations in these regions were significantly different between controls and PTN subjects.

\section{Discussion}

Chronic neuropathic pain is associated with a significant reduction in gray-matter volume within the somatosensory thalamus and reduced blood flow in the thalamic reticular nucleus, the magnitude of which is correlated to pain intensity; i.e., the greater the ongoing pain, the greater the reduction in blood flow. Additionally, subjects with neuropathic pain have reduced thalamic GABA content and display strengthened functional connectivity between the somatosensory thalamus and cerebral cortex, in particular, the primary and secondary somatosensory cortices. These data support the hypothesis that after peripheral nerve injury, loss of thalamic somatosensory neurons results in altered thalamic reticular nucleus activity, a subsequent reduction in GABAergic transmission, and ultimately a change in thalamocortical rhythm, which may result in the constant perception of pain.

The reduction in thalamic volume reported here extends our previous finding in a mixed population of neuropathic pain subjects and confirms previous data in other neuropathic pain conditions (Apkarian et al., 2004; Gustin et al., 2011). We have previously shown that decreased thalamic volume is associated with a decrease $N$-acetyl aspartate/creatine ratio, a reliable marker of neuronal integrity. Consistent with this finding, direct damage to the posterolateral thalamus, a region including the VP, is associated with a considerable risk for the development of chronic neuropathic pain (Kim et al., 2007; Klit et al., 2009; Hong et al., 2010). However, it remains controversial as to whether the development of thalamic stroke pain depends on the lesion including the VP thalamus alone or whether it requires involvement of the posterior part of the ventromedial or ventrocaudalis portae thalamic as well (Gücer et al., 1978; Hendler et al., 1979; Willis et al., 2002).

Although the mechanism responsible for altered VP thalamic volume in neuropathic pain remains unknown, in animal models of neuropathic pain, trans-synaptic apoptosis occurs in the dorsal horn and higher, nociceptive pathway recipient regions, such as the midbrain periaqueductal gray matter (Scholz et al., 2005; Mor et al., 2011). Furthermore, blocking apoptosis with a caspase inhibitor prevents neural loss and attenuates pain behaviors (Scholz et al., 2005). After peripheral nerve injury in humans, pathway-specific neuronal death may occur in areas including the VP thalamus, resulting in the changes in thalamic volume. This volume change does not affect innocuous somatosensory processing because neither the location nor the magnitude of VP activations during innocuous lip brushing was altered in our PTN subjects. We have previously reported that even though the

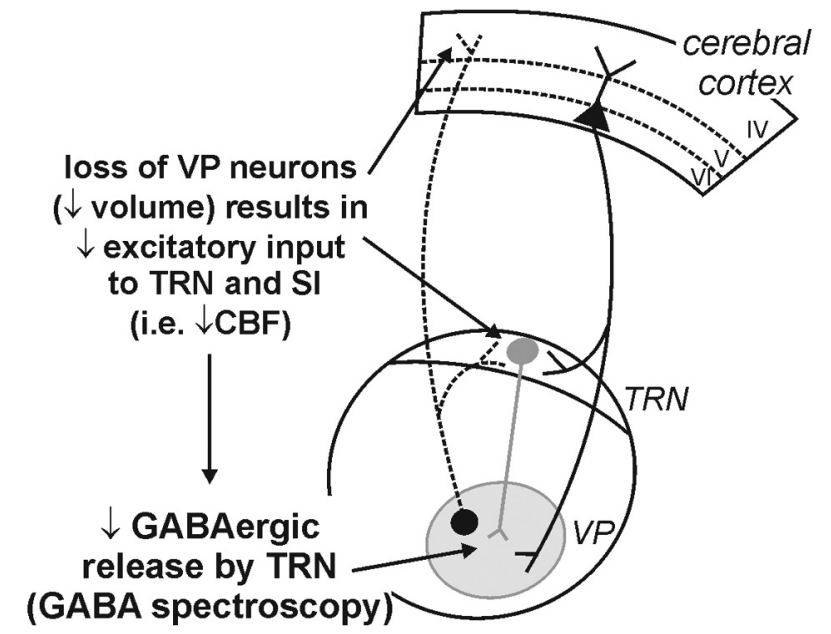

\section{altered thalamocortical connectivity (fMRI connectivity)}

\section{cortex interprets aberrant input as "PAIN"}

Figure 6. Proposed central events which result in the development and/or maintenance of chronic neuropathic pain. An initial loss of neurons in the VP results in a loss of excitatory inputs to the TRN which in turn results in altered inhibitory GABA input in VP thalamus. This disturbed thalamocortical activity may result in the constant perception of pain.

S1 region representing the lip remains unaltered in PTN subjects, the magnitude of S1 activations is reduced (Gustin et al., 2012), although it remains unknown whether acute noxious processing also remains unaffected.

Consistent with the hypothesis that decreased VP thalamic volume results from a loss of VP output neurons, we found that two major VPM recipient regions, the TRN and the orofacial representation of the $\mathrm{S} 1$, displayed reduced ongoing activities in PTN subjects. Within the TRN, reduced blood flow was located in the region shown to contain somatosensory responsive neurons in monkeys and cats, i.e., the anterior lateral region (Pollin and Rokyta, 1982), and within S1, reduced blood flow occurred in the orofacial representation. Positron emission tomography investigations have also reported thalamic blood flow decreases in neuropathic pain subjects, although the precise locations of these decreases are difficult to determine given the spatial resolution of this scanning method (Hsieh et al., 1995; Iadarola et al., 1995).

A detailed picture of TRN organization has begun to emerge in recent times. Most VPM neurons projecting to $\mathrm{S} 1$ send collaterals to TRN GABAergic neurons, and in turn, layer VI neurons in S1 that project to VPM also send collaterals to TRN (Pinault, 2004; Lam and Sherman, 2011). Furthermore, the TRN itself innervates cortically projecting VPM neurons. Given this, a loss of cortically projecting VPM neurons would result in decreased excitatory input to the TRN and S1 and, consequently, a reduction in VPM GABA release due to reduced TRN activation. The decreased thalamic GABA content that occurred in our PTN subjects could result from decreased GABA production in presynaptic terminals or through increased GABA reuptake and catabolism. Although GABA is produced in presynaptic terminals, GABA 
signal includes contributions from different pools of GABA, including that stored in presynaptic vesicles, free GABA in the synaptic cleft, as well as in glial cells after reuptake. Additionally, in the macaque, peripheral nerve lesions result in GABA receptor downregulation (Rausell et al., 1992) and somatosensory pathway lesions reduce GABA-immunoreactive synapses by up to $50 \%$ within the VP thalamus (Ralston, 2005). Although we are the first to report thalamic GABAergic alteration in human neuropathic pain syndromes, indirect evidence for an imbalance in excitatory/inhibitory neurotransmission also comes from the relative success of the GABA-derivative drugs, gabapentin and pregabalin, in the control of neuropathic pain.

More globally, changes in TRN inputs and outputs have a significant effect on overall brain function. Evidence suggests that the TRN regulates all sensory information passing from the thalamus to the cerebral cortex (Pinault, 2004; Lam and Sherman, 2011). Although not conclusive, thalamocortical and TRN neurons also appear to be critical for the production of thalamocortical rhythms with evidence that the intrinsic and network properties of TRN neurons endow them with oscillatory properties (Llinás et al., 1999). For example, Steriade et al. (1985) reported spindle-related rhythms in disconnected TRN neurons and abolition of spindle oscillations following TRN lesion. It has recently emerged that chronic neuropathic pain is associated with significantly altered thalamocortical rhythm (Sarnthein et al., 2006; Walton and Llinás, 2010). In particular neuropathic pain is associated with higher spectral power in the $2-25 \mathrm{~Hz}$ range and a shift of the dominant peak toward lower frequencies (Sarnthein et al., 2006). Furthermore, this altered rhythm is characterized by persistent theta and beta overactivations in painassociated areas, including the primary and secondary somatosensory cortical regions (Stern et al., 2006).

Consistent with these previous reports, we found altered functional connectivity between the VPM thalamus and cortical regions including SI, SII (secondary somatosensory cortex), and anterior insula in our neuropathic pain subjects. Because individuals with neuropathic pain display increased burst firing of VP thalamic neurons, we speculate that periods of high-intensity neuronal firing and intervening low-intensity activity result in enhanced signal covariation between VP thalamus and connected brain regions. We propose that the greater the thalamic GABA decrease, the greater the VP burst firing and the greater the connectivity. This altered connectivity may not be confined to pain processing circuitry, as recently shown in experimental animals that neuropathic pain is associated with impaired spatial working memory and increased spiking phase precision of medial prefrontal-hippocampal circuit with respect to hippocampal theta rhythm (Cardoso-Cruz et al., 2013). Interestingly, this altered fronto-hippocampal connectivity and impaired memory was not associated with a significant change in overall firing rate, just a change in firing rhythm. Similarly, we found no overall increases in CBF, an index of neural activity, in pain-related brain regions, which is consistent with many previous investigations (Hsieh et al., 1995; Wik et al., 2003; Yunus et al., 2004). These data suggest that altered thalamocortical rhythm, and not necessarily increased overall activity in somatosensory processing regions, can result in the persistent perception of pain. A similar situation may also occur in other positive sensory conditions. For example, in individuals with tinnitus, increased auditory intensity is accompanied by decreased medial geniculate nucleus activity which results in reduced auditory cortex inhibition (van Gendt et al., 2012). Similar to chronic neuropathic pain, this pattern of change is thought to be a manifestation of thalamocortical loops dysregulation (Llinás et al., 1999).
Together, our MRI results support the thalamocortical concept of chronic neuropathic pain (Llinás et al., 1999; Jeanmonod et al., 2001; Sarnthein et al., 2006). This model proposes that after nervous system damage, altered afferent input to specific and nonspecific thalamic nuclei results in disturbed thalamocortical circuits involving altered inputs and outputs of cortical somatosensory areas and the thalamic reticular nucleus. Our data show that neuropathic pain is associated with significant neural loss within VP thalamus and that this loss is associated with decreased activity in the TRN and S1 (Fig. 6). As a consequence, GABAergic content of the thalamus is reduced, likely as a direct result of decreased TRN activity. Finally, this reduction in TRN inhibitory output results in altered thalamic firing patterns, altered thalamocortical rhythm, which in some manner, generates the subjective feeling of persistent pain.

These data raise the possibility that prevention of thalamic neuronal loss after peripheral nerve injury, for example, using caspase inhibitors, may prevent the development of chronic neuropathic pain (Scholz et al., 2005). Alternatively, once the pain has been established, altering thalamocortical loops by targeting thalamic lesions, such as to the centrolateral nucleus, may alter thalamocortical processing and relieve chronic pain (Sarnthein et al., 2006). Ultimately, therapeutic interventions aimed at altering thalamocortical loops may provide better outcomes than are currently available for many subjects suffering with persistent neuropathic pain.

\section{References}

Apkarian AV, Sosa Y, Sonty S, Levy RM, Harden RN, Parrish TB, Gitelman DR (2004) Chronic back pain is associated with decreased prefrontal and thalamic gray matter density. J Neurosci 24:10410-10415. CrossRef Medline

Ashburner J, Friston KJ (2005) Unified segmentation. Neuroimage 26:839851. CrossRef Medline

Cardoso-Cruz H, Lima D, Galhardo V (2013) Impaired spatial memory performance in a rat model of neuropathic pain is associated with reduced hippocampus-prefrontal cortex connectivity. J Neurosci 33:2465-2480. CrossRef Medline

Davis KD, Kiss ZH, Tasker RR, Dostrovsky JO (1996) Thalamic stimulation-evoked sensations in chronic pain patients and in nonpain (movement disorder) patients. J Neurophysiol 75:1026-1037. Medline

Di Piero V, Jones AK, Iannotti F, Powell M, Perani D, Lenzi GL, Frackowiak RS (1991) Chronic pain: a PET study of the central effects of percutaneous high cervical cordotomy. Pain 46:9-12. CrossRef Medline

Edden RA, Barker PB (2007) Spatial effects in the detection of gammaaminobutyric acid: improved sensitivity at high fields using inner volume saturation. Magn Reson Med 58:1276-1282. CrossRef Medline

Friston KJ, Holmes AP, Worsley KP, Proline JP, Frith CD, Frackowiak RS (1995) Statistical parametric maps in functional imaging: a general imaging approach. Hum Brain Mapp 2:189-210. CrossRef

Gerke MB, Duggan AW, Xu L, Siddall PJ (2003) Thalamic neuronal activity in rats with mechanical allodynia following contusive spinal cord injury. Neuroscience 117:715-722. CrossRef Medline

Gücer G, Niedermeyer E, Long DM (1978) Thalamic EEG recordings in patients with chronic pain. J Neurol 219:47-61. CrossRef Medline

Gustin SM, Peck CC, Wilcox SL, Nash PG, Murray GM, Henderson LA (2011) Different pain, different brain: thalamic anatomy in neuropathic and non-neuropathic chronic pain syndromes. J Neurosci 31:5956-5964. CrossRef Medline

Gustin SM, Peck CC, Cheney LB, Macey PM, Murray GM, Henderson LA (2012) Pain and plasticity: is chronic pain always associated with somatosensory cortex activity and reorganization? J Neurosci 32:14874-14884. CrossRef Medline

Head H, Holmes G (1911) Sensory disturbances from cerebral lesions. Brain 34:102-254.

Hendler N, Viernstein M, Gucer P, Long D (1979) A preoperative screening test for chronic back pain patients. Psychosomatics 20:801-808. Medline Hirayama T, Dostrovsky JO, Gorecki J, Tasker RR, Lenz FA (1989) Record- 
ings of abnormal activity in patients with deafferentation and central pain. Stereotact Funct Neurosurg 52:120-126. CrossRef Medline

Hong JH, Bai DS, Jeong JY, Choi BY, Chang CH, Kim SH, Ahn SH, Jang SH (2010) Injury of the spino-thalamo-cortical pathway is necessary for central post-stroke pain. Eur Neurol 64:163-168. CrossRef Medline

Hsieh JC, Belfrage M, Stone-Elander S, Hansson P, Ingvar M (1995) Central representation of chronic ongoing neuropathic pain studied by positron emission tomography. Pain 63:225-236. CrossRef Medline

Iadarola MJ, Max MB, Berman KF, Byas-Smith MG, Coghill RC, Gracely RH, Bennett GJ (1995) Unilateral decrease in thalamic activity observed with positron emission tomography in patients with chronic neuropathic pain. Pain 63:55-64. CrossRef Medline

Jeanmonod D, Magnin M, Morel A, Siegemund M, Cancro R, Lanz M (2001) Thalamocortical dysrhythmia II: clinical and surgical aspects. Thalamus Relat Syst 1:245-254. CrossRef

Kim JH, Greenspan JD, Coghill RC, Ohara S, Lenz FA (2007) Lesions limited to the human thalamic principal somatosensory nucleus (ventral caudal) are associated with loss of cold sensations and central pain. J Neurosci 27:4995-5004. CrossRef Medline

Klit H, Finnerup NB, Jensen TS (2009) Central post-stroke pain: clinical characteristics, pathophysiology, and management. Lancet Neurol 8:857-868. CrossRef Medline

Lam YW, Sherman SM (2011) Functional organization of the thalamic input to the thalamic reticular nucleus. J Neurosci 31:6791-6799. CrossRef Medline

Latremoliere A, Woolf CJ (2009) Central sensitization: a generator of pain hypersensitivity by central neural plasticity. J Pain 10:895-926. CrossRef Medline

Lenz FA, Kwan HC, Dostrovsky JO, Tasker RR (1989) Characteristics of the bursting pattern of action potentials that occurs in the thalamus of patients with central pain. Brain Res 496:357-360. CrossRef Medline

Lenz FA, Seike M, Richardson RT, Lin YC, Baker FH, Khoja I, Jaeger CJ, Gracely RH (1993) Thermal and pain sensations evoked by microstimulation in the area of human ventrocaudal nucleus. J Neurophysiol 70:200212. Medline

Lenz FA, Garonzik IM, Zirh TA, Dougherty PM (1998) Neuronal activity in the region of the thalamic principal sensory nucleus (ventralis caudalis) in patients with pain following amputations. Neuroscience 86:1065-1081. CrossRef Medline

Llinás RR, Ribary U, Jeanmonod D, Kronberg E, Mitra PP (1999) Thalamocortical dysrhythmia: a neurological and neuropsychiatric syndrome characterized by magnetoencephalography. Proc Natl Acad Sci U S A 96: 15222-15227. CrossRef Medline

Macey PM, Macey KE, Kumar R, Harper RM (2004) A method for removal of global effects from fMRI time series. Neuroimage 22:360-366. CrossRef Medline

Melzack R (1999) Pain: an overview. Acta Anaesthesiol Scand 43:880-884. CrossRef Medline

Melzack R, Loeser JD (1978) Phantom body pain in paraplegics: evidence for a central "pattern generating mechanism" for pain. Pain 4:195-210. Medline

Mescher M, Merkle H, Kirsch J, Garwood M, Gruetter R (1998) Simultaneous in vivo spectral editing and water suppression. NMR Biomed 11:266272. CrossRef Medline

Moayedi M, Desouza D, Erpelding N (2011) Making sense of gray matter abnormalities in chronic orofacial pain: synthesizing divergent findings. J Neurosci 31:12396-12397. CrossRef Medline

Moisset X, Bouhassira D (2007) Brain imaging of neuropathic pain. Neuroimage 37:S80-S88. CrossRef Medline

Mor D, Bembrick AL, Austin PJ, Keay KA (2011) Evidence for cellular injury in the midbrain of rats following chronic constriction injury of the sciatic nerve. J Chem Neuroanat 41:158-169. CrossRef Medline

Nurmikko TJ, Eldridge PR (2001) Trigeminal neuralgia: pathophysiology, diagnosis and current treatment. Br J Anaesth 87:117-132. CrossRef Medline
Pattany PM, Yezierski RP, Widerström-Noga EG, Bowen BC, MartinezArizala A, Garcia BR, Quencer RM (2002) Proton magnetic resonance spectroscopy of the thalamus in patients with chronic neuropathic pain after spinal cord injury. Am J Neuroradiol 23:901-905. Medline

Petersen ET, Lim T, Golay X (2006) Model-free arterial spin labeling quantification approach for perfusion MRI. Magn Reson Med 55:219-232. CrossRef Medline

Pinault D (2004) The thalamic reticular nucleus: structure, function and concept. Brain Res Brain Res Rev 46:1-31. CrossRef Medline

Pollin B, Rokyta R (1982) Somatotopic organization of nucleus reticularis thalami in chronic awake cats and monkeys. Brain Res 250:211-221. CrossRef Medline

Ralston HJ 3rd (2005) Pain and the primate thalamus. Prog Brain Res 149: 1-10. CrossRef Medline

Rausell E, Cusick CG, Taub E, Jones EG (1992) Chronic deafferentation in monkeys differentially affects nociceptive and nonnociceptive pathways distinguished by specific calcium-binding proteins and down-regulates gamma-aminobutyric acid type A receptors at thalamic levels. Proc Nat Acad Sci U S A 89:2571-2575. CrossRef Medline

Sarnthein J, Stern J, Aufenberg C, Rousson V, Jeanmonod D (2006) Increased EEG power and slowed dominant frequency in patients with neurogenic pain. Brain 129:55-64. CrossRef Medline

Schmidt-Wilcke T, Luerding R, Weigand T, Jurgens T, Schuierer G, Leinisch E, Bogdahn U (2007) Striatal grey matter increase in patients suffering from fibromyalgia: a voxel-based morphometry study. Pain 132:S109-S116. CrossRef Medline

Scholz J, Broom DC, Youn DH, Mills CD, Kohno T, Suter MR, Moore KA, Decosterd I, Coggeshall RE, Woolf CJ (2005) Blocking caspase activity prevents transsynaptic neuronal apoptosis and the loss of inhibition in lamina II of the dorsal horn after peripheral nerve injury. J Neurosci 25:7317-7323. CrossRef Medline

Schweinhardt P, Kuchinad A, Pukall CF, Bushnell MC (2008) Increased gray matter density in young women with chronic vulvar pain. Pain 140: 411-419. CrossRef Medline

Steriade M, Deschênes M, Domich L, Mulle C (1985) Abolition of spindle oscillations in thalamic neurons disconnected from nucleus reticularis thalami. J Neurophysiol 54:1473-1497. Medline

Stern J, Jeanmonod D, Sarnthein J (2006) Persistent EEG overactivation in the cortical pain matrix of neurogenic pain patients. Neuroimage 31:721-731. CrossRef Medline

van Gendt MJ, Boyen K, de Kleine E, Langers DR, van Dijk P (2012) The relation between perception and brain activity in gaze-evoked tinnitus. J Neurosci 32:17528-17539. CrossRef Medline

von Hehn CA, Baron R, Woolf CJ (2012) Deconstructing the neuropathic pain phenotype to reveal neural mechanisms. Neuron 73:638-652. CrossRef Medline

Walton KD, Llinás RR (2010) Central pain as a thalamocortical dysrhythmia: a thalamic efference disconnection? In: Translational pain research: from mouse to man (Kruger L, Light AR, eds). Boca Raton, FL: CRC.

Wik G, Fischer H, Bragée B, Kristianson M, Fredrikson M (2003) Retrosplenial cortical activation in the fibromyalgia syndrome. Neuroreport 14:619-621. CrossRef Medline

Willis WD Jr, Zhang X, Honda CN, Giesler GJ Jr (2002) A critical review of the role of the proposed VMpo nucleus in pain. J Pain 3:79-94. CrossRef Medline

Yezierski RP (1996) Pain following spinal cord injury: the clinical problem and experimental studies. Pain 68:185-194. CrossRef Medline

Younger JW, Shen YF, Goddard G, Mackey SC (2010) Chronic myofascial temporomandibular pain is associated with neural abnormalities in the trigeminal and limbic systems. Pain 149:222-228. CrossRef Medline

Yunus MB, Young CS, Saeed SA, Mountz JM, Aldag JC (2004) Positron emission tomography in patients with fibromyalgia syndrome and healthy controls. Arthritis Rheum 51:513-518. CrossRef Medline 